\title{
Profiles of Surface Mosaics on Chronic Lymphocytic Leukemias Distinguish Stable and Progressive Subtypes
}

\author{
Pauline Y. Huang ${ }^{1}$, Philippa Kohnke ${ }^{1}$, Larissa Belov ${ }^{1}$, O. Giles Best ${ }^{2}$, Stephen P. Mulligan ${ }^{2}$ and Richard I. Christopherson ${ }^{1}$ \\ ${ }^{1}$ School of Molecular Bioscience, University of Sydney, Sydney, NSW 2006, Australia \\ ${ }^{2}$ Northern Blood Research Centre, Kolling Institute of Medical Research, Royal North Shore Hospital, St. Leonards, NSW \\ 2065, Australia
}

Received, April 14, 2013; Revised, May 15, 2013; Accepted, May 22, 2013; Published, May 22, 2013.

\begin{abstract}
Purpose. Chronic lymphocytic leukemia (CLL) is a heterogeneous disease, some patients may survive for many years, while $20-30 \%$ of patients progress and may die within several years. Currently, there is not a single procedure that enables accurate prognosis and triaging of those patients who need immediate and aggressive treatment. All CLL cells are characterised by the expression of the B-cell antigens CD19, CD20, CD21, CD22 and CD23, with aberrant expression of the T-cell antigen CD5. Methods. We have developed a CD antibody microarray (DotScan) containing 182 immobilised CD antibodies that has been used to obtain extensive surface profiles of CLL cells obtained from 96 patients. Results. Of these 182 antigens, 27 were significantly differentially expressed between stable, stable-progressive and progressive CLL. Some of these antigens are not expressed on normal B-cells and may be targets for therapeutic antibodies against CLL. Unsupervised hierarchical clustering of the surface profiles from 96 patients showed that those with progressive CLL could be distinguished based solely upon this 'disease signature'. The sensitivity (proportion of actual positives correctly identified) was $67.9 \%$, the specificity (proportion of negatives correctly identified) was $77.5 \%$, and the accuracy was $71.9 \%$. Conclusions. Considerable effort by a number of research groups has resulted in identification of individual markers for progressive CLL, but their collective use is yet to provide a test that identifies CLL patients at risk. Data presented here provide a basis for development of a simple test using an antibody microarray.
\end{abstract}

This article is open to POST-PUBLICATION REVIEW. Registered readers (see "For Readers") may comment by clicking on ABSTRACT on the issue's contents page.

\section{INTRODUCTION}

Chronic lymphocytic leukemia (CLL) is now considered to be a compartmentalised disease with proliferative and resting compartments, proliferation occurs in the lymph node microenvironment. Cells are released to the peripheral blood where they become resting and accumulate to high levels $\left(200 \times 10^{6}\right.$ cells $\left./ \mathrm{mL}\right)$ constituting a significant metabolic burden to the patient. Only a minor proportion $(<1 \%)$ of circulating CLL cells are proliferating (1), there is strong evidence that the lymph nodes contain proliferating CLL cells that are the primary source of the CLL cells in peripheral blood. The proliferative compartment in lymph nodes is becoming a major focus of therapeutic interest as the highly active B-cell Receptor (BCR) signalling blockers such as ibrutinib (inhibiting Bruton's tyrosine kinase) and idelalisib (blocking PI3-kinase) appear to act by blocking cellular activation in the nodal microenvironment, with major clinical benefits on nodal size, and an early simultaneous lymphocytosis due to lymphocyte redistribution (2).

CLL is more common in people over 50 and carries significant morbidity and mortality. Western countries have a high incidence of CLL, constituting $31 \%$ of all leukemias. With an aging population, economic and societal burdens of CLL will only increase. CLL has an extremely heterogeneous prognosis, with survival times from initial diagnosis as short as 2 years for one-third of patients that have progressive disease. A further one-third of patients will develop progressive disease, while the remaining one third have stable disease that only requires periodic monitoring.

Corresponding Author: Prof Richard I. Christopherson, School of Molecular Bioscience, University of Sydney, Sydney, NSW 2006, Australia. Email: richard.christopherson@sydney.edu.au 
Currently available prognostic markers for progressive CLL include un-mutated genes for the immunoglobulin heavy chain (IGVH), increased levels of ZAP-70, CD38 and activation-induced cytidine deaminase (AID), and the chromosome deletions 11q- and 17p- $(3,4)$. When used collectively, these disease markers do not enable accurate identification of patients with progressive disease, or likely to progress to a terminal phase.

The DotScan CD antibody microarray was developed in our laboratory to enable rapid classification of the common leukaemias, based upon a dot pattern of captured cells with no other information. Live human leukocytes are incubated on the microarray and cells expressing particular $\mathrm{CD}$ antigens on their surface are captured by the corresponding immobilized antibodies. A clinical trial of DotScan was completed with 796 patients and normal subjects using a DotScan microarray containing monoclonal antibodies against $82 \mathrm{CD}$ antigens (5). Statistical analysis of the surface profiles obtained identified disease signatures that showed better than $95 \%$ concordance with diagnoses made using established pathological criteria. In this paper, we have used an extended DotScan microarray containing antibodies against $182 \mathrm{CD}$ antigens to obtain extensive surface profiles of CLL cells from 96 patients. These profiles have been correlated with the known clinical outcomes for the patients as follows; stable (S), slowprogressive (SP), and progressive (P). The data obtained provide the basis for a simple test enabling triaging and vigorous treatment of patients at risk of potentially fatal progressive CLL. The CD antigens that are differentially expressed on progressive CLL are potential targets for therapeutic antibodies, taken either singly or as unusual pairs for interaction with bifunctional engineered antibodies. The area of therapeutic antibodies is the most rapidly growing area of pharmaceuticals.

\section{MATERIALS AND METHODS}

\section{Positive selection of CLL cells from blood samples}

Patient samples (100) were obtained with informed consent and local ethical approval from the Australasian Leukaemia and Lymphoma tissue bank (ALLG), Royal North Shore Hospital (St Leonards, Australia), and the University of Leicester (Leicester, UK). The diagnosis of CLL was made by standard clinical and surface marker criteria (CLL Guide-lines, (6)). The disease status of each patient was assessed and defined as stable (S), slowprogressive (SP) or progressive (P). Of the 100 samples of CLL used in this study, 4 lacked clinical status and were eliminated, 56 were progressive CLL, 23 were slow-progressive CLL, and 17 were stable. Mononuclear cells were obtained from heparinised peripheral blood by Ficoll-Hypaque density gradient centrifugation. Cells were frozen at $-80^{\circ} \mathrm{C}$ in fetal calf serum (FCS) with $10 \%$ dimethyl sulfoxide (DMSO). Samples were thawed and Blymphocytes isolated using magnetic microbeads coated with CD19 antibody. The B-cells were isolated by retention on a MACS LS column (Miltenyi Biotec Inc., Bergisch Gladhach, Germany), and then profiled on DotScan microarrays. This procedure removed contaminating T-cells that could result in incorrect surface profiles for the CLL cells. Only live cells are captured by the immobilized antibodies on DotScan microarrays.

\section{Preparation of microarrays and live CLL cells for surface profiling}

Microarrays were obtained from Medsaic Pty. Ltd. (Sydney, NSW, Australia), and procedures for cell capture, washing, scanning and data analysis were as described by Belov et al. (7). The main section of the DotScan CLL microarray contained duplicate antibodies against $182 \mathrm{CD}$ antigens selected from papers describing the prognostic significance of $C D$ antigens on CLL, and B-cell CD antigens designated at the HLDA9 workshop (Barcelona, Spain, 2010; (8)). A second section of the microarray contained dilutions of isotype control antibodies. The number of viable B-lymphocytes in each sample was determined by trypan-blue exclusion. Viable cells $\left(3 \times 10^{6}\right)$ in $300 \mu \mathrm{L}$ of PBS were applied to DotScan microarrays and incubated for $12 \mathrm{~min}$ at room temperature. Unbound cells were gently washed off using PBS, and the bound cells were fixed with $3.7 \%(\mathrm{w} / \mathrm{v})$ formaldehyde in PBS for 20-30 min, then washed with PBS.

\section{Imaging of patterns of CLL cells captured on microarrays}

Dot patterns of captured cells on microarrays were scanned with a DotReader (Medsaic Pty. Ltd., Sydney, NSW, Australia) providing a digital optical image for analysis with DotScan software. Densities of the dots were quantified on an 8-bit greyness scale (1-256). Any non-specific binding to the equivalent isotype control antibodies was subtracted from the binding values for $\mathrm{CD}$ antibodies. Data 
processed with the DotScan software (Medsaic Pty. Ltd.) were normalized by mean-centred normalization, ensuring that data were normally distributed across all assays. Analysis of variance was run separately for each $\mathrm{CD}$ antibody. Antibodies with ANOVA p-values $<0.05$ after multiple testing correction, using the Benjamini and Hochberg false discovery rate method $(9,10)$, were retained as differentially expressed.

Typical surface profiles for normal leukocytes from peripheral blood, and CLL cells from a patient with progressive disease are shown in Figure 1. These bar charts provide a relative measure of the level of expression of particular $\mathrm{CD}$ antigens, although the height of the bars could also be influenced by the affinity of the antibody-antigen interactions and the relative abundance of particular cell types in a mixed population. Figure la shows a surface profile for the mixed population of peripheral blood mononuclear cells. The bars shown in yellow are T-cell antigens, bars in blue are B-cell antigens, and bars in red are myeloid. The surface profile for purified B-cells (CD19+) from a patient with progressive CLL (Figure 1b) is very different to Figure 1a. CD5 (yellow), normally a Tcell antigen, is aberrantly expressed with the B-cell antigens CD19, CD20, CD21, CD22 and CD23 (all blue). Unusual pairs of $\mathrm{CD}$ antigens, such as CD5 and $\mathrm{CD} 20$, expressed on cancer cells due to mutations could be used as targets for bifunctional antibodies that are now being engineered and tested. The DotScan microarray can be used to identify such unusual pairs of $\mathrm{CD}$ antigens.

\section{RESULTS}

Using an extended DotScan antibody microarray (Version 3, $182 \mathrm{CD}$ antibodies), the following 27 $\mathrm{CD}$ antigens were significantly differentially abundant for progressive CLL (mean intensity $>1.0$ ): CD11a, CD11b, CD11c, CD18, CD19, CD20 (2 antibody clones), CD21, CD22, CD23, CD24, CD25, CD38, CD40, CD43, CD45, CD45RA, CD52, CD69, CD81, CD84, CD98, CD102, CD148, CD180, CD196, and CD270 (Figure 2). The dot intensity data for these $27 \mathrm{CD}$ antigens from 96 patient samples were subjected to a partial least squares discriminant analysis using the statistical Caret $R$ package for estimating the performance of a predictive model. "Leave-one out" cross-validation was used to check the performance of the discriminant analysis using a single observation from the original sample as the validation data, and the remaining observations as the training data. When all $182 \mathrm{CD}$ antibodies were included (isotype control antibodies excluded), the accuracy of clustering samples according to clinical status was $71.9 \%$ with a $95 \%$ confidence interval of $62-81 \%$. The sensitivity (i.e., proportion of actual positives correctly identified) was $67.9 \%$, and the specificity (i.e., proportion of negatives correctly identified) was $77.5 \%$. This analysis indicates that the extensive surface profiles obtained provide a basis for triaging progressive CLL patients requiring immediate treatment. An additional 10 surface molecules were differentially abundant but were below the threshold intensity of 1.0 (see Figure 2).

\section{DISCUSSION}

Of the 37 differentially abundant $\mathrm{CD}$ antigens shown in Figure 2, 9 are coloured blue for progressive CLL in the heatmap indicating high levels: CD19, CD20, CD21, CD23, CD40, CD43, CD45, CD45RA and CD148. The empirical use of patterns of $\mathrm{CD}$ antigens should enable discrimination between subtypes of CLL, but the functions of these antigens in the biology of progressive CLL are also important. The functions of most of these antigens correlate in some way with proliferation or cell adhesion as summarized in Table 1.

The surface profiles of differentially abundant membrane proteins on cells for progressive, stableprogressive or stable CLL could be used as 'disease signatures' for diagnosis and triaging of patients that are at risk. However, the accuracy of this procedure is at present only $72 \%$ which is not sufficiently high to be used as a routine pathology test. A higher level of discrimination is required between the S, SP and P subtypes of CLL. A future Version 4 of the DotScan CLL microarray would include antibodies against additional differentially abundant CD antigens. Some of the antibodies in the current Version 3 microarray that did not contribute to discrimination between CLL subtypes would be eliminated. We have recently completed a project on identification of differentially abundant proteins from stable, stable-progressive and progressive CLL using isobaric tags for absolute and relative quantitation (iTRAQ) with analysis of tryptic peptides by two-dimensional liquid chromatography (2DLC) and identification and 

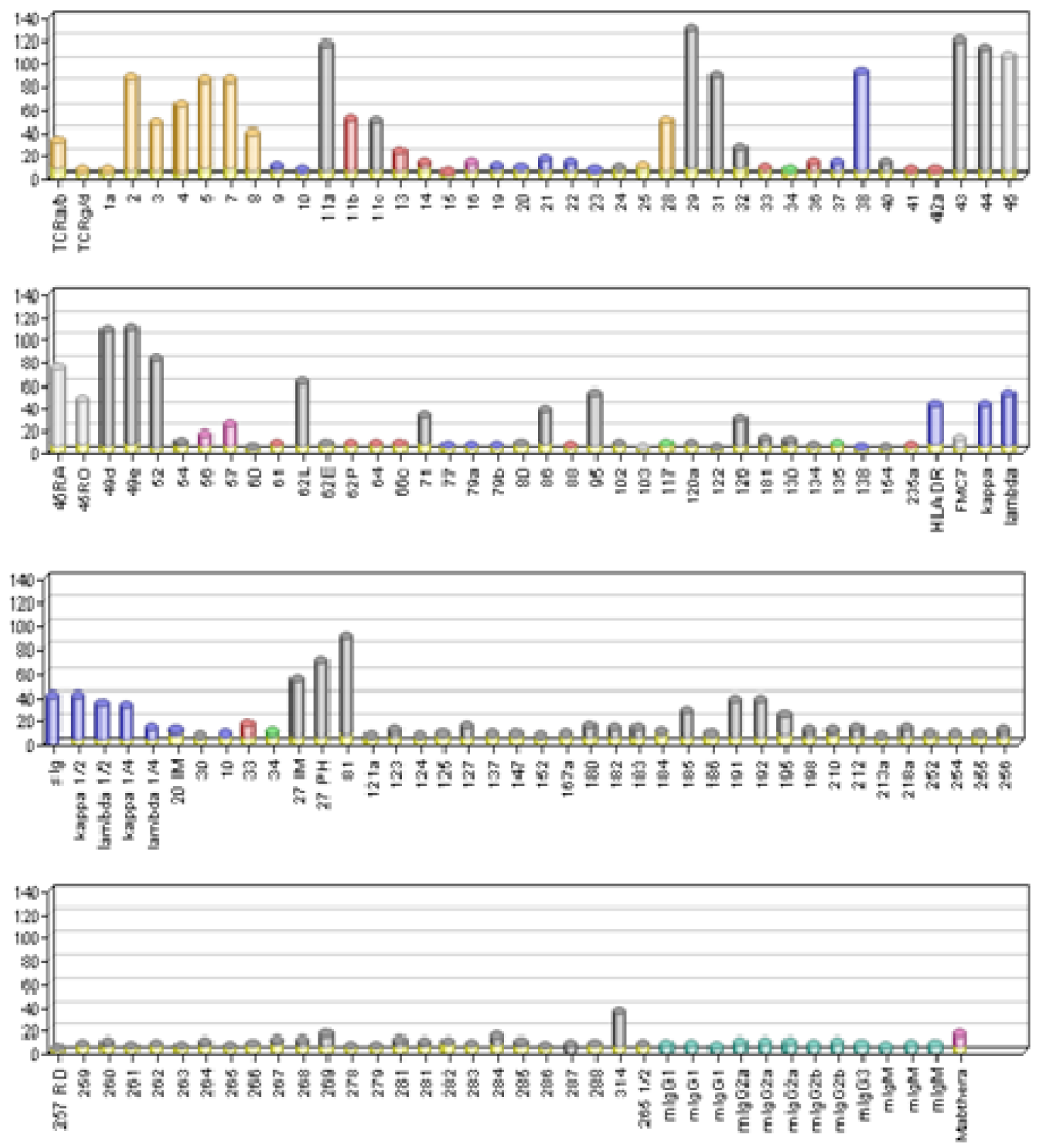

(a) Normal leukocytes

quantitation of proteins using tandem mass spectrometry (MS/MS; Huang, Christopherson et al., unpublished). This procedure of iTRAQ-2DLCMS/MS enables discovery of new protein markers that could be included as antibodies in a Version 4 of a DotScan CLL microarray. We hope that further development of DotScan (Version 4) will lead to a microarray suitable for routine determination of subtypes of CLL in samples of peripheral blood.

\section{CONCLUSION}

The repertoires of surface molecules (CD antigens) are different on different subtypes of CLL, reflecting changes in the genetic programs of cells determined by mutations. 

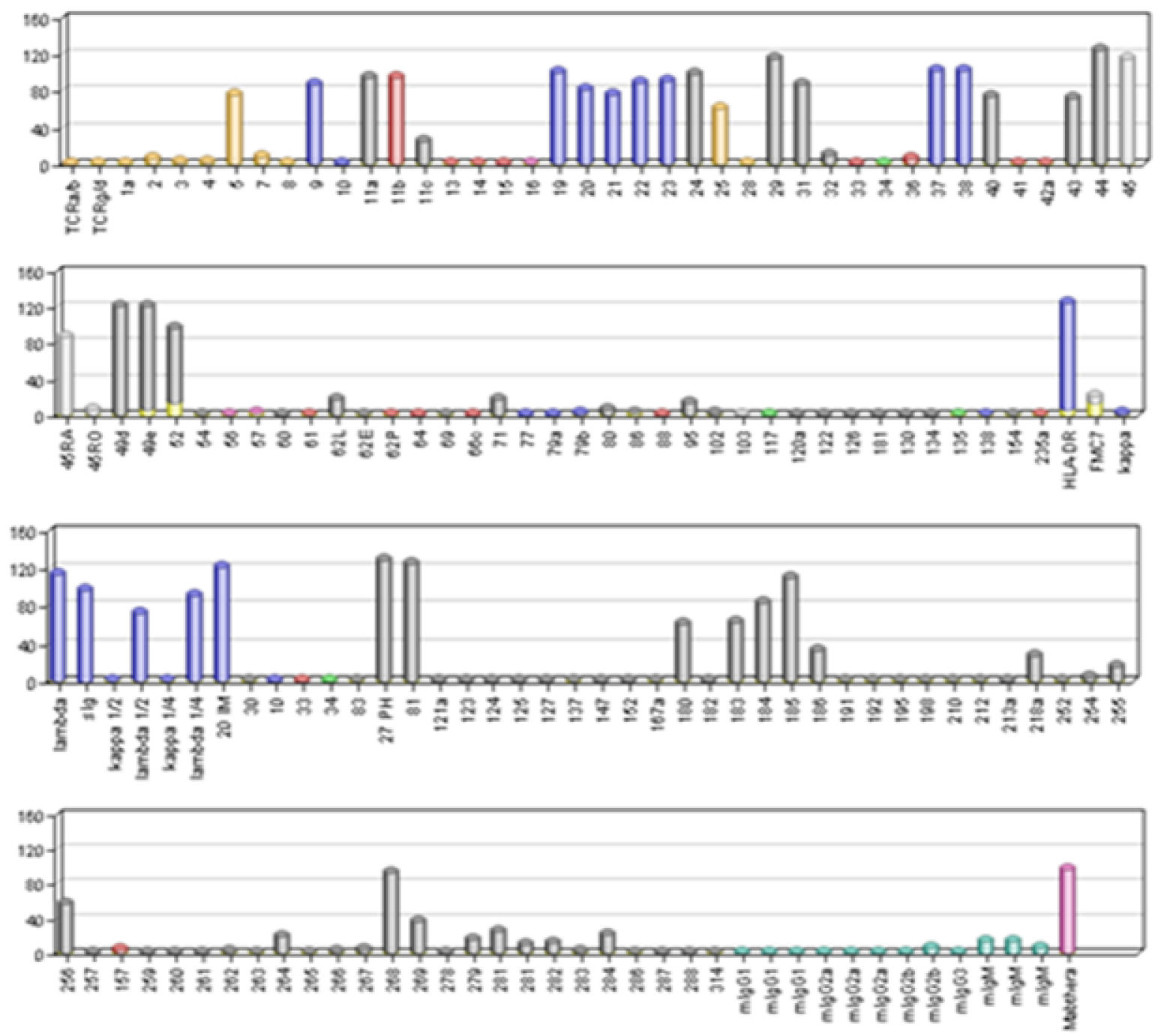

\section{(b) Progressive CLL cells}

Figure 1. Surface profiles of CD antigens on normal leukocytes and progressive CLL cells. (a) Mononuclear leukocytes from a normal subject; (b) CLL cells purified by positive selection on CD19 antibody-coated Miltenyi magnetic beads. The procedures used are described in Materials and Methods. Cell intensity is shown on the ordinate, and CD antigen number on the abscissa.

Extensive surface profiles may be used as disease signatures; rather than being 'too much information' they have the potential to triage CLL patients with high accuracy. Cultured human cells could also be used to screen natural products as toxins or allergens by identification of induced changes in surface profiles. Such changes could be determined using appropriate DotScan CD antibody microarrays. In Europe, recent legislation regulates the use of animal models for chemical testing, and this type of in vitro testing could provide a suitable alternative (12).

\section{CONFLICT OF INTEREST}

The authors have no conflict of interest to declare.

\section{ACKNOWLEDGEMENTS}

This research was supported by a grant from the Leukemia Foundation of Australia and the Chronic Lymphocytic Leukemia Global Research Foundation. 


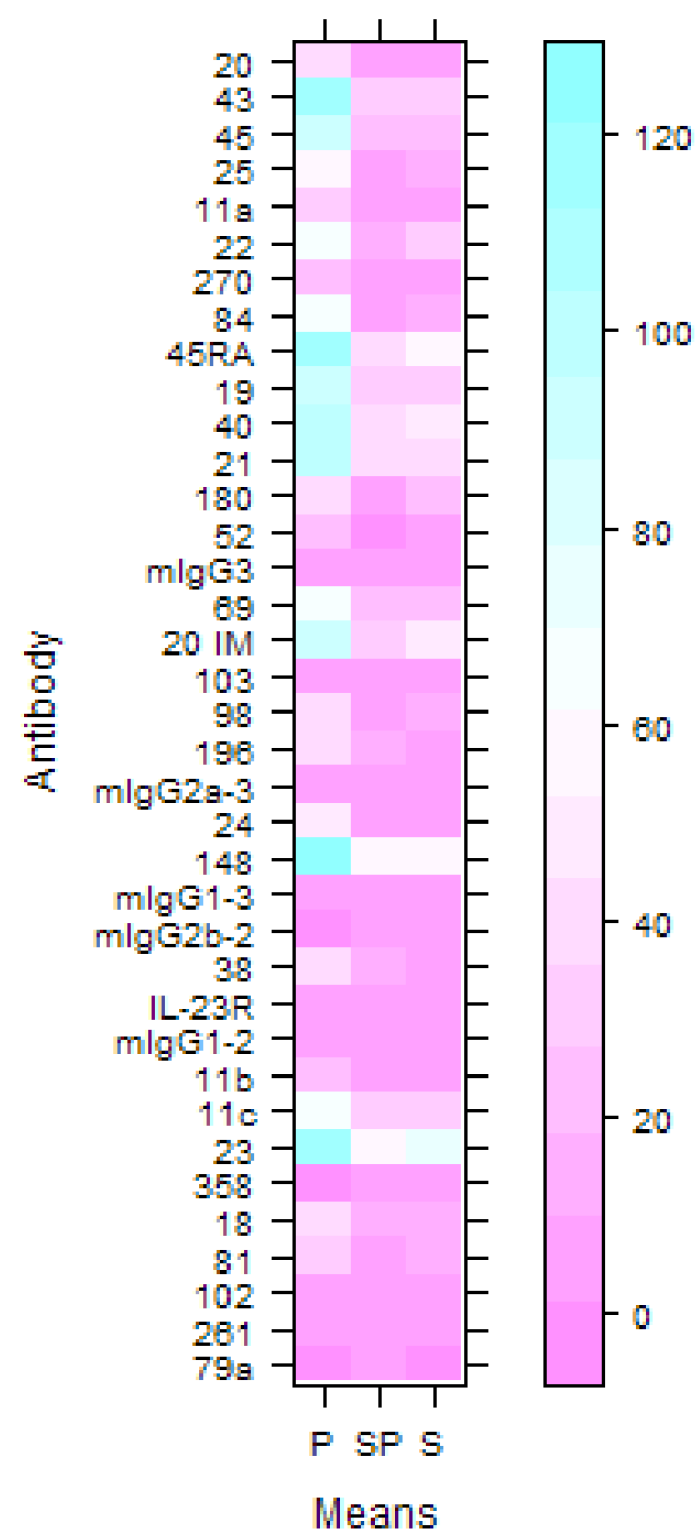

Figure 2. Surface signatures of CD antigens for progressive CLL (P), versus slow-progressive (SP, and stable (S) CLL. The 37 differentially abundant $\mathrm{CD}$ antigens (surface molecules) are aligned vertically and colour coded according to the bar on the right to indicate relative intensities (Means) of cells captured on these antibody dots. The mean expression levels were quantified using an 8-bit pixel greyness scale from 1-256 for each differentially abundant antigen between the 3 clinical groups (P, SP and S) of collectively 96 CLL patients.

\begin{tabular}{ll}
\hline Table 1. Functions of CD antigens up-regulated on progressive CLL (11). \\
\hline CD antigen & Function \\
\hline CD19 & Forms part of B-cell receptor (BCR) complex with CD21 and CD81. \\
CD20 & Forms calcium channel, regulator of cell cycle progression and B-cell activation and \\
& proliferation. \\
CD21 & Part of B-cell antigen receptor complex, involved with B-cell activation. \\
CD23 & Low affinity IgE receptor. \\
CD40 & TNF receptor 5, receptor for CD154 (CD40L), co-stimulatory signal to B-cells. \\
CD43 & Inhibits cell-cell adhesion of leukocytes. \\
CD45 & Receptor-like protein tyrosine phosphatase, B-cell antigen receptor-mediated activation \\
CD45RA & Contains exon 4 with alternative splicing, otherwise as for CD45. \\
CD148 & Tyrosine phosphatase, binding antibody leads to cytokine secretion. \\
\hline
\end{tabular}




\section{REFERENCES}

1. Messmer BT, Messmer D, Allen SL, Kolitz JE, Kudalkar P, Cesar D, Murphy EJ, Koduru P, Ferrarini M, Zupo S, Cutrona G, Damle RN, Wasil T, Rai KR, Hellerstein MK, Chiorazzi N. In vivo measurements document the dynamic cellular kinetics of chronic lymphocytic leukemia B cells. J Clin Invest, 2005; 115:755-64.

2. Wiestner A. Emerging role of kinase-targeted strategies in chronic lymphocytic leukemia, Blood, 2012; 120:4684-4691.

3. Badoux XC, Keating MJ, Wierda WG. What is the best frontline therapy for patients with CLL and $17 \mathrm{p}$ deletion? Curr Hematol Malig Rep, 2011; 6:36-46.

4. Huang PY, Best OG, Belov L, Mulligan SP, Christopherson RI. Surface profiles for subclassification of chronic lymphocytic leukemia. Leuk Lymphoma, 2012; 53:1046-56.

5. Belov L, Mulligan SP, Barber N, Woolfson A, Scott M, Stoner K, Chrisp JS, Sewell WA, Bradstock KF, Bendall L, Pascovici DS, Thomas M, Erber W, Huang P, Sartor M, Young GA, Wiley JS, Juneja S, Wierda WG, Green AR, Keating MJ, Christopherson RI. Analysis of human leukaemias and lymphomas using extensive immunophenotypes from an antibody microarray. Br J Haematol, 2006; 135:184-97.

6. Hallek M, Cheson BD, Catovsky D, CaligarisCappio F, Dighiero G, Döhner H, Hillmen P, Keating MJ, Montserrat E, Rai KR, Kipps TJ.
International Workshop on Chronic Lymphocytic Leukemia. Guidelines for the diagnosis and treatment of chronic lymphocytic leukemia: a report from the International Workshop on Chronic Lymphocytic Leukemia updating the National Cancer Institute-Working Group 1996 guidelines. Blood, 2008; 111:5446-56.

7. Belov L, Huang P, Barber N, Mulligan SP, Christopherson RI. Identification of repertoires of surface antigens on leukemias using an antibody microarray. Proteomics, 2003; 3:2147-2154.

8. Matesanz-Isabel J, Sintes J, Llinàs L, de Salort J, Lázaro A, Engel P. New B-cell CD molecules. Immunol Lett, 2011; 134:104-12.

9. Hamelinck D, Zhou H, Li L, Verweij C, Dillon D, Feng Z, Costa J, Haab BB. Optimized normalization for antibody microarrays and application to serum-protein profiling. Mol Cell Proteomics, 2005; 4:773-784.

10. Sill M, Schroder C, Hoheisel JD, Benner A, Zucknick M. Assessment and optimisation of normalisation methods for dual-colour antibody microarrays. BMC Bioinformatics, 2010; 11:556.

11. Zola H, Swart B, Nicholson I, Voss E. Leukocyte and stromal cell molecules: The CD markers. John Wiley and Sons Inc. New Jersey, USA, 2007.

12. Johansson H, Albrekt AS, Borrebaeck CA, Lindstedt M. The GARD assay for assessment of chemical skin sensitizers. Toxicol In Vitro, 2013; 27:1163-9. 